\title{
Robust trapped-ion quantum logic gates by continuous dynamical decoupling
}

\author{
A. Bermudez, ${ }^{1}$ P. O. Schmidt ${ }^{2}$ M. B. Plenio, ${ }^{1}$ and A. Retzker ${ }^{1}$ \\ ${ }^{1}$ Institut für Theoretische Physik, Albert-Einstein Allee 11, Universität Ulm, 89069 Ulm, Germany \\ ${ }^{2}$ QUEST Institute, Physikalisch-Technische Bundesanstalt and Leibniz University Hannover, 38116 Braunschweig, Germany
}

(Received 20 October 2011; published 4 April 2012)

\begin{abstract}
We introduce a scheme that combines phonon-mediated quantum logic gates in trapped ions with the benefits of continuous dynamical decoupling. We demonstrate theoretically that a strong driving of the qubit decouples it from external magnetic-field noise, enhancing the fidelity of two-qubit quantum gates. Moreover, the scheme does not require ground-state cooling, and is inherently robust to undesired ac Stark shifts. The underlying mechanism can be extended to a variety of other systems where a strong driving protects the quantum coherence of the qubits without compromising the two-qubit couplings.
\end{abstract}

DOI: 10.1103/PhysRevA.85.040302

PACS number(s): 03.67.Lx, 32.80.Qk, 37.10.Ty

A quantum processor is an isolated quantum device where information can be stored quantum-mechanically over long periods of time, but can be also manipulated and retrieved. This forbids its perfect isolation, making such a device sensitive to the noise introduced by either external sources, or experimental imperfections. Additionally, the interactions between distant quantum bits (qubits), as required to perform quantum logic operations, are frequently achieved by auxiliary (quasi)particles whose fluctuations introduce an additional source of noise. As emphasized recently [1], one of the big challenges of quantum-information science is the quest for methods to cope with all these natural error sources, achieving error rates that allow fault-tolerant quantum error correction.

We address this problem for trapped atomic ions [2]. Among the most relevant sources of noise in this system [3], we can list the following: (i) thermal noise introduced by auxiliary phonons, (ii) fluctuating external magnetic fields, (iii) uncompensated ac Stark shifts due to fluctuations in the laser parameters, and (iv) drifts in the phases of the applied laser beams. There are two different strategies to overcome these obstacles: (a) Minimize the thermal fluctuations by laser cooling [4], searching for gates operating faster than the time scale set by the other noise sources [5], or (b) look for schemes that are intrinsically robust to the noise. Among the latter, there are schemes that provide partial solutions, such as the gates robust to the thermal ion motion [6,7], or the encoding in magnetic-field-insensitive states [8] and decoherence-free subspaces [9]. Recently, there has been a growing effort to implement microwave-based quantum-information processing [10-12], exploiting the excellent control over the phase and amplitude of microwaves as compared to laser fields. Despite these efforts, it remains a key challenge to suppress all of the above sources of noise. Here, we propose to accomplish such a step, achieving fault-tolerant error bounds, by a continuous version of dynamical decoupling at reach of current technology.

While pulsed dynamical decoupling is a well-developed technique [13], already demonstrated for ions [14], its optimal combination with two-qubit gates requires a considerable additional effort [15]. Hence, simpler protocols are a subject of recent interest $[11,16]$. We hereby present a decoupling scheme well suited, but not limited, to trapped-ion experiments with three important properties: generality, simplicity, and robustness. It is sufficiently general to be applied to any type of ion qubits. It is simple since it combines two standard tools, namely, a carrier and a red-sideband excitation. In particular, it relies on the strong driving of the carrier transition, which may be realized by laser beams for optical qubits, or by microwaves for hyperfine and Zeeman qubits. With this independent driving source, we improve simultaneously the performance and the speed of the gate, as compared to the light-shift gates [17]. Besides, this driving has the potential of simplifying certain aspects of previous gate schemes [18], and is responsible for the gate robustness at different levels.

The system. We focus on ${ }^{25} \mathrm{Mg}^{+}$to exploit the benefits of microwave technology [19], although the scheme is also valid for other species. Our qubit consists of two hyperfine states $|0\rangle,|1\rangle$ with energy difference $\omega_{0}$ [see Fig. 1(a) and Table I]. The ions form a string along the axis of a linear Paul trap with radial and axial frequencies $\omega_{x}, \omega_{z}$. The small radial vibrations yield a set of vibrational modes of frequencies $\omega_{n}$, whose excitations are the transverse phonons $a_{n}, a_{n}^{\dagger}$ [18]. If the qubitqubit couplings are mediated by these quasiparticles [20], the scheme becomes less sensitive to ion heating or thermal motion, and it is easier to operate within the Lamb-Dicke regime.

As shown in Fig. 1(a), a pair of laser beams in a Raman configuration induces a transition between the qubit states. By setting their frequency beatnote $\omega_{\mathrm{L}}$ close to $\omega_{0}-\omega_{n}$, such that the detuning $\delta_{n}=\omega_{\mathrm{L}}-\left(\omega_{0}-\omega_{n}\right)$ is much smaller than the radial trap frequency [see Table I for the bare detuning $\delta_{\mathrm{L}}=$ $\left.\omega_{\mathrm{L}}-\left(\omega_{0}-\omega_{x}\right)\right]$, one obtains the red-sideband excitation. In addition, we drive the carrier transition. For our particular qubit choice, this driving can be performed with microwave radiation of frequency $\omega_{\mathrm{d}}$, such that $H_{\mathrm{t}}=H_{\mathrm{c}}+H_{\mathrm{r}}$ is

$$
H_{\mathrm{t}}=\sum_{i} \frac{\Omega_{\mathrm{d}}}{2} \sigma_{i}^{+} e^{-i\left(\omega_{\mathrm{d}}-\omega_{0}\right) t}+\sum_{i n} \mathcal{F}_{i n} \sigma_{i}^{+} a_{n} e^{-i \delta_{n} t}+\text { H.c. }
$$

where we have introduced the microwave Rabi frequency $\Omega_{\mathrm{d}}$, and the sideband coupling strengths $\left|\mathcal{F}_{i n}\right| \propto \Omega_{\mathrm{L}} \eta$ scale linearly with the laser Rabi frequency $\Omega_{\mathrm{L}}$ and Lamb-Dicke parameter $\eta$, such that $\left|\mathcal{F}_{i n}\right| \ll \delta_{n}$ (Table I). Here, we use the spin operators $\sigma_{i}^{+}=\left|1_{i}\right\rangle\left\langle 0_{i}\right|$, and we work in the interaction picture rotating with the phonon and qubit frequencies.

Sideband gates and thermal noise. We first introduce an intuitive picture. The Hamiltonian (1) for $\Omega_{\mathrm{d}}=0$ combines 
TABLE I. Specific values of the trapped-ion setup.

\begin{tabular}{|c|c|c|c|c|c|c|c|}
\hline$\omega_{0} / 2 \pi$ & $\omega_{x} / 2 \pi$ & $\omega_{z} / 2 \pi$ & $\eta$ & $\left|\delta_{\mathrm{L}}\right| / 2 \pi$ & $\Omega_{L} / 2 \pi$ & $\Omega_{\mathrm{d}} / 2 \pi$ & $B_{0}$ \\
\hline $1.8 \mathrm{GHz}$ & $4 \mathrm{MHz}$ & $1 \mathrm{MHz}$ & 0.2 & $800 \mathrm{kHz}$ & $500 \mathrm{kHz}$ & $5.2 \mathrm{MHz}$ & $4 \mathrm{mT}$ \\
\hline
\end{tabular}

two noncommuting spin-dependent forces. Each one aims at displacing the normal modes along a closed trajectory in phase space determined by the eigenstates $\left| \pm_{x}\right\rangle,\left| \pm_{y}\right\rangle$ of $\sigma^{x}, \sigma^{y}$ [18]. In Fig. 1(b), we describe the action of the $\sigma^{x}$ force on a single ion [6,7]. Depending on the state $\left| \pm_{x}\right\rangle$, the ion follows a different path in phase space, and after returning to the starting point, it picks a geometric phase only determined by the enclosed area, and independent of the motional state. In contrast, for a single red sideband, $\sigma_{x}$ and $\sigma_{y}$ forces are implemented, resulting in rotations around two orthogonal axis [Fig. 1(c)]. In a Trotter decomposition, the concatenation of these orthogonal displacements spoils the closing of the trajectory, such that the qubit and phonon states remain entangled. As shown below, this makes the gate sensitive to thermal fluctuations.
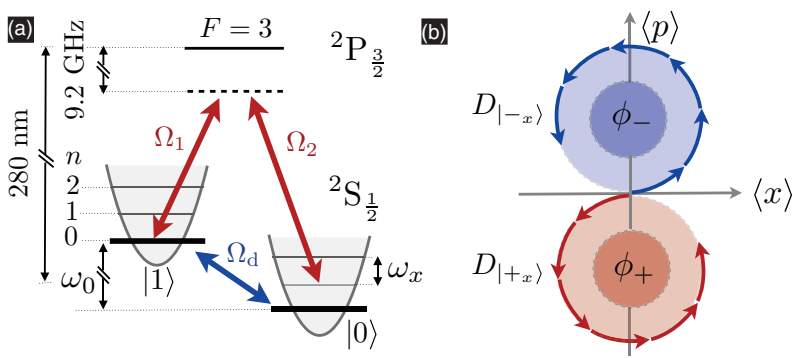

(c)
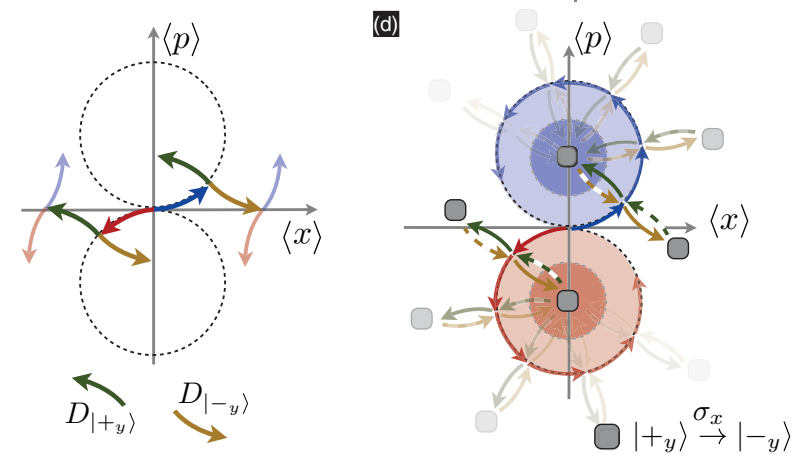

FIG. 1. (Color online) (a) Hyperfine structure of ${ }^{25} \mathrm{Mg}^{+}$. The states $|0\rangle=\left|F=3, m_{F}=3\right\rangle$ and $|1\rangle=|2,2\rangle$ form our qubit. Two laser beams $\Omega_{1}, \Omega_{2}$ drive the red sideband via an off-resonant excited state, and a microwave $\Omega_{\mathrm{d}}$ directly couples to the transition. (b) Spin-dependent $\sigma^{x}$ force acting on a single trapped ion. The phonons associated with the states $\left|+_{x}\right\rangle,\left|-_{x}\right\rangle$ are displaced in phase space according to $D_{\left| \pm_{x}\right\rangle}$, and form different closed paths that lead to the geometric phases $\phi_{ \pm}$. (c) Trotterization of the combined $\sigma^{x}$ and $\sigma^{y}$ forces. The $\sigma^{x}$ displacement $D_{|+x\rangle}$ shall be followed by the two possible $\sigma^{y}$ displacements $D_{\left| \pm_{y}\right\rangle}$ since $\left|+_{x}\right\rangle \propto\left(\left|+_{y}\right\rangle+i\left|-{ }_{y}\right\rangle\right.$. Hence, the phase-space trajectory is not generally closed. (d) Schematic spin-echo refocusing of the $\sigma^{y}$ force. By applying a $\pi$-pulse $X_{i}^{\pi}=\sigma_{i}^{x}$ (box) at half the $\sigma^{y}$ displacements, one obtains $\left| \pm_{y}\right\rangle \rightarrow\left|\mp_{y}\right\rangle$, such that the displacements are reversed (dotted arrows), and the trajectory is refocused, yielding a well-defined geometric phase.
For large detunings $\left|\delta_{\mathrm{L}}\right| \gg \Omega_{\mathrm{L}} \eta$, the lasers only excite virtually the vibrational modes, and the phonons can be adiabatically eliminated. In fact, it is the process where a phonon is virtually created by an ion, and then reabsorbed by a distant one, which gives rise to the effective $X Y$ couplings [21]

$$
H_{\mathrm{eff}}=\sum_{i j} J_{i j}^{\mathrm{eff}} \sigma_{i}^{+} \sigma_{j}^{-}, \quad J_{i j}^{\mathrm{eff}}=-\sum_{n} \frac{1}{\delta_{n}} \mathcal{F}_{i n} \mathcal{F}_{j n}^{*} .
$$

At certain instants of time, the unitary evolution corresponds to a SWAP gate [22], which performs the logic operation $\left|1_{i} 0_{j}\right\rangle \leftrightarrow$ $\left|0_{i} 1_{j}\right\rangle$ while leaving the remaining states unchanged. However, there is an additional process that spoils the performance of the gate, namely, the phonon might be reabsorbed by the same ion. This leads to a residual spin-phonon coupling

$$
H_{\mathrm{res}}=\sum_{i} \hat{B}_{i}(t) \sigma_{i}^{z}, \quad \hat{B}_{i}(t)=\sum_{n m} B_{i n m} a_{m}^{\dagger} a_{n} e^{-i\left(\omega_{n}-\omega_{m}\right) t},
$$

where $B_{i n m}=-\frac{1}{2} \mathcal{F}_{i n} \mathcal{F}_{i m}^{*}\left(\frac{1}{\delta_{n}}+\frac{1}{\delta_{m}}\right)$. Accordingly, the resonance frequency fluctuates in time due to the motional dynamics, leading to a local thermal noise in the limit of many ions that introduces dephasing. In Fig. 2(a), the critical effect of this term on the SWAP gate is displayed. We compare the numerical simulation of the full spin-phonon Hamiltonian to the effective idealized description (2). As evidenced in this figure, the gate performance is severely modified by the thermal phonon ensemble. As the mean phonon number is increased, the oscillations get a stronger damping, and the generation of Bell states at half the SWAP periods (arrows) deteriorates.

Achieving robustness against thermal noise. We now show how to protect the coherent spin dynamics from this thermal dephasing by switching $\Omega_{\mathrm{d}} \neq 0$. Schematically, this may be
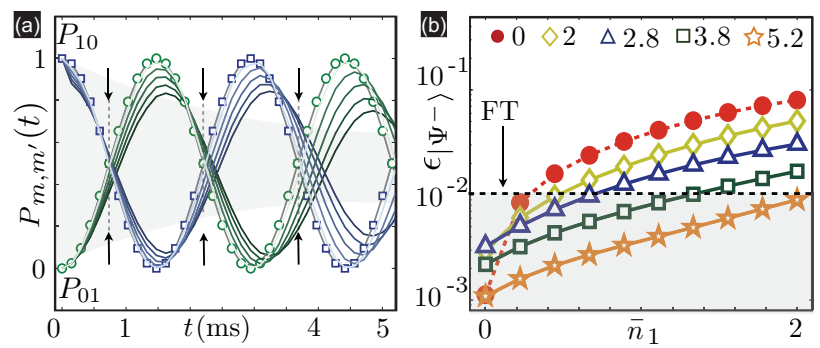

FIG. 2. (Color online) (a) Dynamics of the swap probabilities $P_{10}(t)$ (squares) and $P_{01}(t)$ (circles) for the effective gate (2), compared to the exact spin-phonon Hamiltonian for a two-ion crystal with $\bar{n}=\{0,0.1,1,2,4\}$ (solid lines). The phonon Hilbert space is truncated to $n_{\max }=20$ excitations per mode. (b) Error $\epsilon=1-\mathcal{F}$ for the generation of the Bell state $\left|\Psi^{-}\right\rangle$as a function of the mean phonon number and $n_{\max }=14$. As the driving is increased, $\Omega_{\mathrm{d}} / \omega_{z} \in\{0,2,3.8,5.2\}$, the error lies within the fault-tolerance (FT) threshold. 
accomplished by refocusing one of the spin-dependent forces using a series of spin-echo pulses [23] that invert the atomic state [Fig. 1(d)]. We show that the strong driving of the carrier transition implements a continuous version of this refocusing, providing a viable mechanism for overcoming this noise. As will become clear later on, this driving also minimizes the undesired errors due to ac Stark shifts and magnetic-field noise.

A helpful account of the decoupling mechanism may be the following. In the dressed-state basis of the driving $\left| \pm_{x}\right\rangle_{i}=$ $\left(\left|1_{i}\right\rangle \pm\left|0_{i}\right\rangle\right) / \sqrt{2}$, the residual spin-phonon coupling becomes $H_{\text {res }}(t)=\sum_{i n m} B_{i n m}\left|+_{x}\right\rangle_{i}\left\langle{ }_{x}\right| a_{m}^{\dagger} a_{n} e^{i\left[\Omega_{\mathrm{d}}-\left(\omega_{n}-\omega_{m}\right)\right] t}+$ H.c. For a strong driving strength $\Omega_{\mathrm{d}}$, this term rotates very fast even for two vibrational modes that are close in frequency, and can be thus neglected in a rotating-wave approximation. Note that the same argument applies for any stable driving phase [18].

This simple argument has to be readdressed for a combination of the carrier and red-sideband interactions (1), since the residual couplings are no longer described by Eq. (3). In order to show that a similar argument can still be applied, we have performed a polaron-type transformation [18]. We find that the dynamics is described by the effective Hamiltonian

$$
\tilde{H}_{\mathrm{eff}}=\sum_{i j} \tilde{J}_{i j}^{\mathrm{eff}} \sigma_{i}^{x} \sigma_{j}^{x}+\frac{1}{2} \sum_{i} \Omega_{\mathrm{d}} \sigma_{i}^{x}, \quad \tilde{J}_{i j}^{\mathrm{eff}}=\frac{1}{4} J_{i j}^{\mathrm{eff}},
$$

whereas the residual spin-phonon coupling is given by

$$
\tilde{H}_{\mathrm{res}}=\sum_{i n} \frac{i}{2}\left(\mathcal{F}_{i n} a_{n}-\mathcal{F}_{i n}^{*} a_{n}^{\dagger}\right)\left(\cosh \hat{\Theta}_{i} \sigma_{i}^{y}-i \sinh \hat{\Theta}_{i} \sigma_{i}^{z}\right) \text {, }
$$

such that $\hat{\Theta}_{i}=\sum_{m}\left(\mathcal{F}_{i m} a_{m}-\mathcal{F}_{i m}^{*} a_{m}^{\dagger}\right) / 2 \delta_{m}$. By moving to the dressed-state basis, the residual term only involves transitions between the dressed eigenstates $\left|+_{x}\right\rangle \leftrightarrow\left|-_{x}\right\rangle$, supplemented by the transformation on the phonons encoded in the different powers of $\hat{\Theta}_{i}$. Fortunately, all these transitions are inhibited due to the large energy gap between the dressed states set by $\Omega_{\mathrm{d}}$. More precisely, in the strong driving regime $\Omega_{\mathrm{d}} \gg 2 \delta_{n}$ (see Table I), the leading-order terms of the residual coupling (5) can be neglected in a rotating-wave approximation.

To check the correctness of this argument, we integrate numerically the complete Hamiltonian (1), and take into account the thermal motion of the trapped ions. After the unitary evolution $U\left(t_{\mathrm{f}}\right)=U\left(t_{\mathrm{f}}, \frac{1}{2} t_{\mathrm{f}} t\right)\left(\sigma_{i}^{z} \sigma_{j}^{z}\right) U\left(\frac{1}{2} t_{\mathrm{f}}, 0\right)$, we calculate the fidelity of producing the Bell state $\left|\Psi^{-}\right\rangle=(|10\rangle-i|01\rangle) / \sqrt{2}$, $\mathcal{F}_{|\Psi-\rangle}=\max _{t_{\mathrm{f}}}\left\{\left\langle\Psi^{-}\left|\operatorname{Tr}_{\mathrm{ph}}\left[U\left(t_{\mathrm{f}}\right) \rho_{0} U^{\dagger}\left(t_{\mathrm{f}}\right)\right]\right| \Psi^{-}\right\rangle\right\}$. The results displayed in Fig. 2(b) demonstrate the promised decoupling from the thermal noise, where one observes that the fidelity of the gate improves considerably when $\Omega_{\mathrm{d}} \gg 2 \delta_{n}$. For driving strengths in the $4-5 \mathrm{MHz}$ range, the gate error lies within the fault-tolerance threshold $\epsilon_{\mathrm{t}} \sim 10^{-2}-10^{-4}[24]$ for states with mean phonon numbers $\bar{n} \leqslant 2$. However, we expect a lower fidelity when experimental imperfections are considered.

In Fig. 3, we describe the experimental steps required to create the desired Bell state. In the first step, the qubits are optically pumped to $|0\rangle$, and the initial state $\left|\psi_{0}\right\rangle=|10\rangle$ is prepared by a $\pi$-pulse $X_{1}^{\pi}$ obtained from a microwave resonant with the carrier transition. Three comments are now in order. First, a magnetic field $B_{0} \approx 4 \mathrm{mT}$ needs to

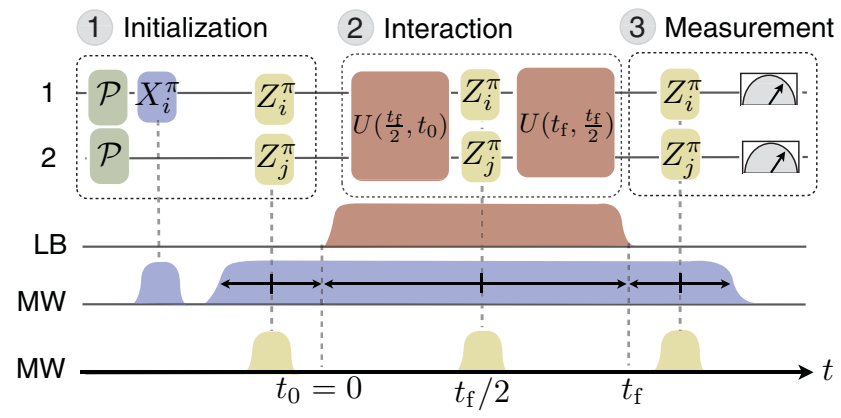

FIG. 3. (Color online) The two upper rows represent the circuit model for the two qubits, and the lower rows represent the switching of the laser beams (LB) and microwaves (MW). The initialization consists of optical pumping $\mathcal{P}$, followed by a local $\pi$-pulse $X_{i}^{\pi}=\sigma_{i}^{x}$. Global $\pi$-pulses $Z_{i}^{\pi}=\sigma_{i}^{z}$ correct possible synchronization errors. The two-qubit coupling is applied by switching on the red sideband (LB), and the carrier (MW) yields the noise decoupling.

be applied to ensure a sufficiently large Zeeman splitting between magnetic states to avoid driving unwanted transitions. Note that the motional excitation due to the microwave is negligible owing to the vanishing Lamb-Dicke factor. Second, either the ac Stark shift from an off-resonant laser beam or a magnetic-field gradient is required to effectively hide the second ion. Alternatively, one could use ion shuttling techniques [25]. Third, we account for the worst possible scenario by considering (i) different switching times of the lasers and microwaves, and (ii) imperfect timing with the microwave. By introducing global $\pi$-pulses $Z_{1}^{\pi} Z_{2}^{\pi}$ from the energy shift of an off-resonant strong microwave, we refocus the fast oscillations caused by the resonant microwave and correct the possible difference of switching times. In the second step, the two-qubit coupling is applied at $t=t_{0}$ by switching on the laser beams responsible for the red sideband. Again, a refocusing pulse at $t=t_{\mathrm{f}} / 2$ shall correct for the imperfect synchronization. In the final step, after switching off the laser and microwaves at $t=t_{\mathrm{f}}$, the qubit state is measured by state-dependent fluorescence techniques. If the announced decoupling has worked correctly, this two-qubit gate should have generated the entangled Bell state $\left|\Psi^{-}\right\rangle$regardless of the phonon state. Let us emphasize that this gate is capable of producing the remaining Bell states by choosing different initial states [18] and, together with single-qubit rotations, becomes universal for quantum computation.

Resilience to magnetic-field noise and ac-Stark shifts. So far, we have neglected the effects of the environment. In standard traps, the leading source of noise is due to environmental fluctuating magnetic fields, which limit the coherence times of magnetic-field-sensitive states to milliseconds [3]. This is particularly important for multi-ion entangled states [26], and will also play a role in our scheme considering that $\tilde{J}_{\text {eff }} / 2 \pi \approx 1 \mathrm{kHz}$. We model the global magnetic-field noise by a fluctuating resonance frequency $H_{\mathrm{n}}=\frac{1}{2} \sum_{i} F(t) \sigma_{i}^{z}$. Here, $F(t)$ is a stochastic Markov process [27] characterized by a diffusion constant $c$ and a correlation time $\tau$, which leads to $\left\langle\sigma^{x}(t)\right\rangle=e^{-t / T_{2}}$ with $T_{2}=2 / c \tau^{2}$ [18]. By fixing these parameters, we can reproduce the experimentally observed $T_{2} \approx$ $5 \mathrm{~ms}$ [Fig. 4(a)], and study its consequences on the two-qubit 

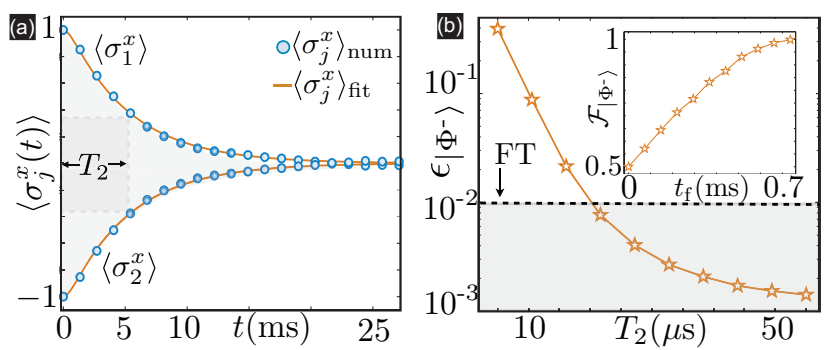

FIG. 4. (Color online) (a) Exponential decay of the coherences $\left\langle\sigma^{x}(t)\right\rangle$ for the initial states $\left|\psi_{0}\right\rangle=\left| \pm_{x}\right\rangle$. The circles represent the numerical statistical average of the $N=5 \times 10^{3}$ evolutions, and the solid line represents the exponential fit. (b) Error in the generation of the Bell state $\left|\Phi^{-}\right\rangle$for the additional magnetic-field noise, together with a strong driving $\Omega_{\mathrm{d}} / 2 \pi=5.2 \mathrm{MHz}$. The error is presented as a function of the $T_{2}$ time. In the inset, we represent the evolution of the fidelity for the typical noise dephasing time $T_{2}=5 f \mathrm{~ms}$.

entangling gate. Notice that the evolution within the zeromagnetization subspace is not affected by this global noise, which is a decoherence-free subspace. Hence, we have studied the fidelity for the Bell state $\left|\Phi^{-}\right\rangle=(|11\rangle-i|00\rangle) / \sqrt{2}$.

The strong driving $\Omega_{\mathrm{d}}$ protects the qubit coherences from this magnetic noise without compromising the entangling gate, and may be considered as a continuous version [11,28] of the so-called dynamical decoupling [13]. To single out the effects of the noise from those of the thermal motion, we have considered a ground-state cooled crystal, setting $\Omega_{\mathrm{d}} / 2 \pi=5.2 \mathrm{MHz}$ to ensure that the results can be carried out to higher temperatures [Fig. 2(b)]. We evaluate numerically the fidelity of generating the Bell state $\left|\Phi^{-}\right\rangle$by averaging over different samplings of the random noise [inset of Fig. 4(b)]. Due to the decoupling, the fidelity approaches unity at the gate time $t_{\mathrm{f}}=0.7 \mathrm{~ms}$. In the main panel of Fig. 4(b), we show that the gate error for shorter coherence times still lies below the fault-tolerance threshold, which demonstrates that the decoupling mechanism supports a stronger magnetic noise. Alternatively, this tells us that the gate tolerates smaller speeds, and thus lower Rabi frequencies of the Raman beams. This shall reduce even further the thermal error studied above, and the spontaneous scattering of photons due to the Raman configuration. We have also calculated the fidelity of the quantum channel with respect to the desired quantum gate $U_{\text {eff }}=e^{-i \frac{\pi}{4} \sigma_{i}^{x} \sigma_{j}^{x}}[18]$, which also lies within the fault-tolerance threshold.

As an additional advantage of our scheme, we note that it also minimizes the effects of uncompensated ac Stark shifts. In the implementations of the geometric phase gates, the shifts caused by off-resonant transitions to all possible states must be compensated by tuning the laser intensities, frequencies, and polarizations [29]. However, fluctuations of these parameters will introduce additional noise. In contrast, these energy shifts are canceled in our scheme by the strong driving, in analogy to the minimization of fluctuating Zeeman shifts. Let us finally comment on the effect of phase instabilities on the gate. In contrast to Mølmer-Sørensen (MS) gates [6], our scheme does not depend on the slow drift of the laser phases. The secondorder process, whereby a phonon is virtually excited and then reabsorbed, is associated with the creation and subsequent annihilation of photons in the same pair of Raman beams, giving rise to the insensitivity to slow changes of the phase [see Eq. (2)]. In contrast, the MS scheme involves two different pairs of Raman beams, such that the crosstalk leads to the phase sensitivity. Hence, our gate (4) only relies on the phase of the microwave, which is easier to stabilize as compared to the phase of the laser beams. We note that concatenated drivings may overcome amplitude fluctuations [30].

Conclusions. We have introduced a scheme that merges continuous dynamical decoupling with warm quantum gates in trapped ions. The decoupling, on top of reducing the magnetic noise, is also responsible for the robustness with respect to thermal fluctuations and ac Stark shifts. Although we have focused on sideband interactions, our scheme could also protect MS gates from magnetic-field fluctuations and ac Stark shifts. Moreover, these ideas may find applications in other architectures that use a bosonic data bus to couple distant qubits, such as superconducting qubits coupled to transmission lines, or color centers in nanodiamonds coupled to nanomechanical resonators.

Note added. Recently we became aware of the interest in similar ideas for atoms in thermal cavities [31] and decoherence-free states in ion traps [32].

Acknowledgment. We are supported by HIP, PICC, AQUTE, QESSENCE, QUEST, and by the Alexander von Humboldt Foundation.
[1] T. D. Ladd et al., Nature (London) 464, 45 (2010).

[2] R. Blatt et al., Nature (London) 453, 1008 (2008).

[3] D. J. Wineland et al., J. Res. Natl. Inst. Stand. Technol. 103, 259 (1998); D. Leibfried et al., Rev. Mod. Phys. 75, 281 (2003); H. Haeffner et al., Phys. Rep. 469, 155 (2008).

[4] J. I. Cirac and P. Zoller, Phys. Rev. Lett. 74, 4091 (1995); C. Monroe, D. M. Meekhof, B. E. King, W. M. Itano, and D. J. Wineland, ibid. 75, 4714 (1995); F. Schmidt-Kaler et al., Nature (London) 422, 408 (2003).

[5] A. Steane et al., Phys. Rev. A 62, 042305 (2000); J. J. GarciaRipoll, P. Zoller, and J. I. Cirac, Phys. Rev. Lett. 91, 157901 (2003); L.-M. Duan, ibid. 93, 100502 (2004).
[6] A. Sørensen and K. Molmer, Phys. Rev. Lett. 82, 1971 (1999); J. Benhelm et al., Nat. Phys. 4, 463 (2008).

[7] G. J. Milburn et al., Fortschr. Phys. 48, 801 (2000); D. Leibfried et al., Nature (London) 422, 412 (2003).

[8] J. J. Bollinger et al., IEEE Trans. Instrum. Meas. 40, 126 (1991); C. Langer et al., Phys. Rev. Lett. 95, 060502 (2005); P. C. Haljan et al., Phys. Rev. A 72, 062316 (2005).

[9] D. A. Lidar, I. L. Chuang, and K. B. Whaley, Phys. Rev. Lett. 81, 2594 (1998); D. Kielpinski et al., Science 291, 1013 (2001); T. Monz et al., Phys. Rev. Lett. 103, 200503 (2009).

[10] F. Mintert and C. Wunderlich, Phys. Rev. Lett. 87, 257904 (2001); A. Khromova et al., e-print arXiv:1112.5302. 
[11] N. Timoney et al., Nature (London) 476, 185 (2011).

[12] C. Ospelkaus et al., Phys. Rev. Lett. 101, 090502 (2008); Nature (London) 476, 181 (2011).

[13] L. Viola, E. Knill, and S. Lloyd, Phys. Rev. Lett. 82, 2417 (1999); L. Viola, S. Lloyd, and E. Knill, ibid. 83, 4888 (1999).

[14] M. Biercuk et al., Nature (London) 458, 996 (2009); D. J. Szwer et al., J. Phys. B 44, 02550 (2011).

[15] S. Montangero, T. Calarco, and R. Fazio, Phys. Rev. Lett. 99, 170501 (2007).

[16] P. Rabl et al., Phys. Rev. B 79, 041302(R) (2009).

[17] D. Jonathan, M. B. Plenio, and P. L. Knight, Phys. Rev. A 62, 042307 (2000); D. Jonathan and M. B. Plenio, Phys. Rev. Lett. 87, 127901 (2001).

[18] See Supplemental Material at http://link.aps.org/supplemental/ 10.1103/PhysRevA.85.040302 for a detailed description of the technical aspects of this work, and for a comparison to other existing gate schemes.

[19] B. Hemmerling et al., Appl. Phys. B 104, 583 (2011).

[20] D. Porras and J. I. Cirac, Phys. Rev. Lett. 92, 207901 (2004); S.-L. Zhu, C. Monroe, and L.-M. Duan, ibid. 97, 050505 (2006).
[21] E. H. Lieb et al., Ann. Phys. 16, 407 (1961); A. Bermudez et al., New J. Phys. 12, 123016 (2010).

[22] M. A. Nielsen and I. L. Chuang, Quantum Computation and Quantum Information (Cambridge University Press, Cambridge, UK, 2004).

[23] E. L. Hahn, Phys. Rev. 80, 580 (1950).

[24] A. M. Steane, Phys. Rev. A 68, 042322 (2003).

[25] D. Kielpinski et al., Nature (London) 417, 709 (2002).

[26] T. Monz et al., Phys. Rev. Lett. 106, 130506 (2011).

[27] D. T. Gillespie, Am. J. Phys. 64, 3 (1996).

[28] P. Facchi and S. Pascazio, Phys. Rev. Lett. 89, 080401 (2002); K. M. Fonseca-Romero, S. Kohler, and P. Hanggi, ibid. 95, 140502 (2005).

[29] D. J. Wineland et al., Philos. Trans. R. Soc., A 361, 1349 (2003).

[30] J.-M. Cai, F. Jelezko, M. B. Plenio, and A. Retzker, e-print arXiv:1111.0930.

[31] S.-B. Zheng, Phys. Rev. A 66, 060303(R) (2002).

[32] A. Noguchi, S. Haze, K. Toyoda, and S. Urabe, Phys. Rev. Lett. 108, 060503 (2012). 